\title{
RETRATO DE LA PROVINCIA JESUÍTICA DE ARAGÓN EL AÑO DESPUÉS DE SU EXTINCIÓN*
}

\author{
POR \\ CARLOS A. MARTínEZ TORNERO \\ Universidad de Alicante \\ martineztornero@gmail.com
}

\section{RESUMEN}

En este trabajo pretendemos reconstruir el estado en el que se encontraba la Provincia jesuítica de Aragón entre dos fechas de gran importancia, como fueron el momento de la expulsión (1767) y el año siguiente al de su extinción canónica (1774). Asimismo, aportaremos información sobre cuestiones como la de los jesuitas secularizados, los que quedaron depositados en España, los novicios que comenzaron a cobrar la pensión vitalicia, etc., para obtener, con todo ello, un retrato aproximado sobre la que fuera Provincia jesuita de Aragón en 1774.

PALABRAS CLAVE: Provincia de Aragón, Compañía de Jesús, expulsión, extinción.

\section{PORTRAIT OF JESUIT PROVINCE OF ARAGON THE YEAR AFTER EXTINCTION}

\begin{abstract}
In this essay we will try to approach to the situation of the Aragon Jesuit Province, between two very important events in the History of the Society of Jesus: the expulsion of the Spanish Kingdom (1767) and the year after the extinction of the Society (1774). Moreover, we will pay attention to some questions like the number of Jesuits that left the Society, those who remained in Spain in 1767, the number of novices that started to get paid after the extinction... and with all this information we will obtain an approached picture about the situation of the Aragon Jesuit Province in 1774.
\end{abstract}

KEY WORDS: Aragon Jesuit Province, Society of Jesus, expulsion, extinction.

Recibido/Received 06-04-2011

Aceptado/Accepted 14-07-2015

\section{INTRODUCCIÓN}

Desde el momento en el que Carlos III se decidió a llevar a cabo la expulsión de la Compañía de Jesús de sus inmensos dominios, esta orden religiosa inició un proceso de declive que se tradujo en una constante disminución en lo que al número de sus integrantes se refiere. Esta fase de desgaste se debió, fundamentalmente, a dos factores: en primer lugar, el número de los jesuitas desterrados se vio reducido por la muerte paulatina de algunos de sus miembros. No debemos olvidar que una buena parte de los expulsos se encontraba en una edad bastante avanzada para la época cuando se les intimó el decreto de expulsión. En concreto, en la provincia de Aragón aproximadamente un quinto de los expulsos superaba los cincuenta años de edad en $1767 .{ }^{1}$
En segundo lugar, la secularización, es decir, el abandono de la Orden por parte de algunos jesuitas, fue la otra gran causa por la que el número de ignacianos se vio reducido.

En este sentido, existe constancia de las presiones a las que fueron sometidos los religiosos para que saliesen de la Orden. Los jueces comisionados en la expulsión, es decir, los funcionarios reales encargados de llevar a la práctica el decreto de destierro de los jesuitas, inducían a los religiosos indecisos hacia la secularización, y en el caso de los novicios las coacciones estuvieron más presentes, amenazándoles incluso con que si decidían continuar al lado de los ignacianos cometerían tres pecados mortales: infidelidad al rey, desobediencia a la Real Pragmática y ausencia de caridad para consigo mismo. ${ }^{2}$

Fernández Arrillaga, I. 2002. "Los novicios de la Compañía de Jesús: la disyuntiva ante el autoexilio y su estancia en Italia", en E. Giménez (ed.), $Y$ en el tercero perecerán. Gloria, caída y exilio de los jesuitas españoles en el siglo XVIII: 251-278, Alicante: Publicaciones de la Universidad de Alicante. 
Todavía hoy no se conoce con exactitud el número de los jesuitas que integraban la Asistencia de España de la Compañía de Jesús en el momento de su expulsión, si bien, lo más probable es que su cifra superase los cinco mil sujetos. En este sentido, resultan ilustrativas las cantidades ofrecidas por diversos investigadores en la materia: Enrique Giménez López y Mario Martínez Gomis hablan de 5.046 individuos, ${ }^{3}$ José A. Ferrer Benimeli calcula 5.152 jesuitas, ${ }^{4}$ la cifra de Teófanes Egido es de 5.376 religiosos $^{5}$ y, finalmente, Ludovico Pastor habla de 5.444 sujetos. ${ }^{6}$ En el presente estudio pretendemos ofrecer una serie de datos relativos a los miembros de la Compañía de Jesús que pertenecían a la Provincia jesuítica de Aragón, un territorio que comprendía las inmediaciones de las actuales comunidades autónomas de Aragón, Cataluña, Valencia y Baleares, con el objetivo de comprobar la evolución de los integrantes de esta demarcación geográfica desde el momento de su salida del reino hispano, en 1767 , hasta el año siguiente a la extinción canónica de la Orden por Clemente XIV, ${ }^{7}$ es decir, hasta 1774.

De esta manera, el primero de los aspectos que nos interesa es el de conocer el número de los religiosos integrantes de la Provincia de Aragón. Para tratar de establecer con exactitud esta cifra, hemos consultado fuentes diversas de archivos tan dispares como el Archivum Romanum Societatis lesu (ARSI), ${ }^{8}$ Archivo del Ministerio de Asuntos Exteriores (AMAAEE), ${ }^{9}$ Arxiu Históric de la Companyia de

3 Giménez López, E. y Martínez Gomis, M. 1997. “La secularización de los jesuitas expulsos (1767-1773)", en E. Giménez (coord.), Expulsión y exilio de los jesuitas españoles: 259-304, Alicante: Publicaciones de la Universidad de Alicante.

4 Ferrer Benimeli, J. A. 1996. La expulsión y extinción de los jesuitas según la correspondencia diplomática francesa, vol. II: 10 y 238, Zaragoza: Universidad de Zaragoza y Universidad Tachira.

5 Egido, T. 1979. "La expulsión de los jesuitas de España”, en R. García Villoslada (coord.), Historia de la Iglesia en España, vol. IV: 745792, Madrid: Biblioteca de Autores Cristianos.

6 Pastor, L. 1954. Storia dei Papi, vol. XVI, I: 754, Roma: Desclée A.C. Editori Pontifici.

7 Sobre la extinción de la Compañía de Jesús véase: Giménez López, Enrique 2008. Misión en Roma. Floridablanca y la extinción de los jesuitas. Murcia: Universidad de Murcia; Giménez López, Enrique 2002. "la Compañía de Jesús (1767-1769)”, en E. Giménez (ed.), Y en el tercero perecerán. Gloria, caída y exilio de los jesuitas españoles en el s. XVIII: 163-193. Alicante: Universidad de Alicante; Giménez López, Enrique: “La extirpación de la mala doctrina: los primeros pasos para la extinción de la Compañía de Jesús", en E. Martínez Ruiz y M. de Pazzis Pi Corrales (coords.), Dogmatismo e intolerancia: 257-274. Madrid: Editorial Actas.

8 En este archivo han resultado de gran ayuda diferentes catálogos como ARSI, Hispaniae, 147, que contiene una versión del número de los jesuitas que pertenecían a las cuatro provincias metropolitanas (Castilla, Toledo, Andalucía y Aragón) en 1767; ARSI, Hispaniae, 149, que recoge una relación nominal de los religiosos de las once provincias hispanas; ARSI, Hispaniae, 150, con una catalogación que resulta ilustrativa en cuanto al número de jesuitas secularizados

9 En la sección Santa Sede existen una serie de legajos dedicados a los auxilios económicos que recibían los jesuitas, bajo la denominación "Socorros a jesuitas" (legajos 550-580). Entre esta documentación se encuentran diferentes listados en los que se recogen por colegios los jesuitas de cada provincia, indicando su lugar de residencia en el momento en el que percibían su pensión, y datos como su muerte, secularización u otros aspectos relacionados con la estancia italiana de los expulsos.
Jesus de Catalunya (AHSIC) ${ }^{10}$ o Archivo General de Simancas (AGS). ${ }^{11}$

Esta primera cuestión, que en un principio podría parecer sencilla, en realidad no lo es tanto, debido a que los diferentes catálogos que hemos encontrado en los archivos señalados no siempre coinciden, y a causa también de las dificultades que existen para rastrear el número de novicios, ya que generalmente no aparecen en las relaciones. Sin embargo, después de consultar las fuentes mencionadas, consideramos que la Provincia jesuítica de Aragón se encontraba integrada por unos 630 efectivos, que se hallaban distribuidos entre los 35 colegios y residencias que formaban esta demarcación territorial. A este número habría que añadir, al menos, 30 novicios que decidieron seguir a la Compañía de Jesús en su destierro. Por lo tanto, estaríamos hablando de 660 religiosos, que se hallaban distribuidos de la manera que se observa en el gráfico que incluimos a continuación:

\section{Jesuitas de LA PRovincia de ARAgón en 1767 POR COLEGIOS ${ }^{12}$}

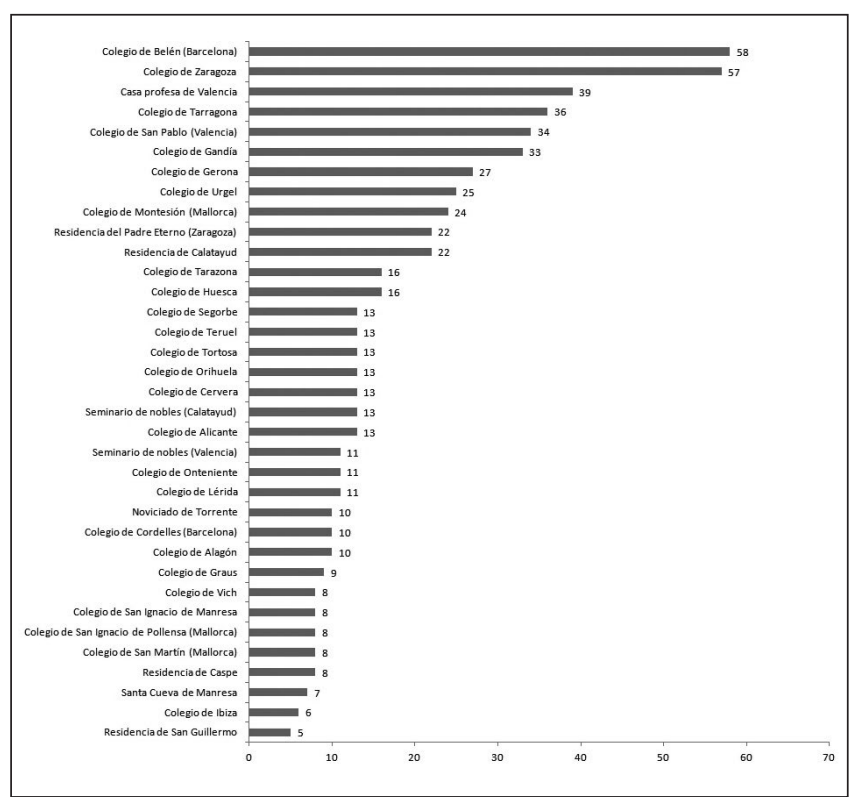

10 De gran importancia ha resultado el catálogo elaborado por la Contaduría general de temporalidades, con fecha de 20 de octubre de 1777, a cuyo frente se encontraba Juan Antonio Archimbaud, en el que se da cuenta de los jesuitas expulsos y se ofrecen datos relativos a su muerte o secularización. Sobre la Contaduría general de temporalidades y sobre el resto de organismos que se generaron después de la expulsión de los jesuitas véase: Martínez Tornero, C. A. 2010. Carlos III y los bienes de los jesuitas: la gestión de las temporalidades por la monarquía borbónica (1767-1815). Alicante: Publicaciones de la Universidad de Alicante.

11 En la sección Marina, el legajo 724 ofrece información relativa al embarque de los jesuitas para su traslado a los Estados Pontificios, y en esos listados se distingue el grado y el colegio al que pertenecían, así como también aparecen otros datos interesantes para nuestro cometido. Dentro de este archivo también resulta de gran interés el inventario 27 de la sección Dirección General del Tesoro.

12 Fuente: Elaboración propia a partir de la información contenida en AHSIC, EX. 22 fols. 81-89; ARSI, Hisp. 147; ARSI, Hisp. 149 y AMAAEE, Santa Sede, leg. 550. 
Como se puede apreciar, los colegios con un mayor número de religiosos eran el colegio de Belén de Barcelona, que contaba con 58 jesuitas (de los cuales 32 eran sacerdotes, 14 coadjutores y 12 escolares), y el colegio de Zaragoza, donde residían 57 individuos (35 sacerdotes y 22 coadjutores). Los colegios con menor importancia en cuanto a su número de integrantes eran la residencia de San Guillermo, en la provincia de Lleida, con 5 jesuitas ( 2 sacerdotes y 3 coadjutores), y el colegio de Ibiza, con 6 ignacianos (3 sacerdotes y 3 coadjutores).

Si prestamos atención al grado de los religiosos en el momento de su expulsión obtendremos los gráficos siguientes:
GRAdo dE LOS JESUITAS DE LA PROVINCIA DE ARAGÓN EN 1767

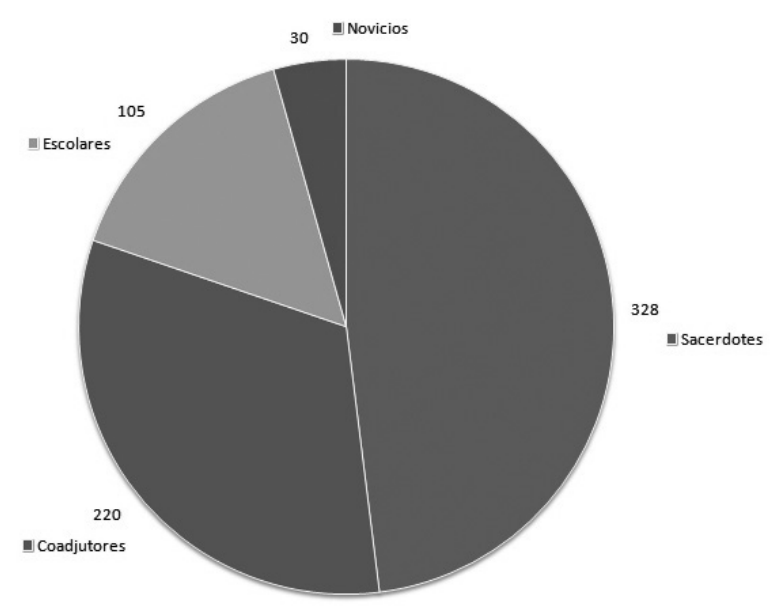

NÚMERO Y GRADO DE JESUITAS POR COLEGIOS EN 1767 (PROVINCIA DE ARAGÓN)

\begin{tabular}{|c|c|c|c|c|}
\hline Colegio & Sacerdotes & Coadjutores & Escolares & Total \\
\hline Colegio de Belén (Barcelona) & 32 & 14 & 12 & 58 \\
\hline Colegio de Zaragoza & 35 & 22 & 0 & 57 \\
\hline Casa Profesa de Valencia & 22 & 17 & 0 & 39 \\
\hline Colegio de Tarragona & 9 & 11 & 16 & 36 \\
\hline Colegio de San Pablo (Valencia) & 11 & 11 & 12 & 34 \\
\hline Colegio de Gandía & 13 & 10 & 10 & 33 \\
\hline Colegio de Gerona & 12 & 8 & 7 & 27 \\
\hline Colegio de Urgel & 9 & 8 & 8 & 25 \\
\hline Colegio de Montesión (Mallorca) & 11 & 10 & 3 & 24 \\
\hline Colegio de Calatayud & 9 & 6 & 7 & 22 \\
\hline Residencia del P. Eterno (Zaragoza) & 15 & 7 & 0 & 22 \\
\hline Colegio de Huesca & 7 & 7 & 2 & 16 \\
\hline Colegio de Tarazona & 10 & 6 & 0 & 16 \\
\hline Colegio de Alicante & 10 & 3 & 0 & 13 \\
\hline Seminario de Nobles (Calatayud) & 8 & 5 & 0 & 13 \\
\hline Colegio de Cervera & 9 & 4 & 0 & 13 \\
\hline Colegio de Orihuela & 8 & 5 & 0 & 13 \\
\hline Colegio de Tortosa & 8 & 5 & 0 & 13 \\
\hline Colegio de Teruel & 9 & 4 & 0 & 13 \\
\hline Colegio de Segorbe & 7 & 5 & 1 & 13 \\
\hline Colegio de Lérida & 8 & 3 & 0 & 11 \\
\hline Colegio de Onteniente & 6 & 4 & 1 & 11 \\
\hline Seminario de Nobles (Valencia) & 6 & 5 & 0 & 11 \\
\hline Colegio de Alagón & 7 & 3 & 0 & 10 \\
\hline Colegio de Cordelles (Barcelona) & 7 & 3 & 0 & 10 \\
\hline Noviciado de Torrente & 3 & 7 & 0 & 10 \\
\hline Colegio de Graus & 4 & 3 & 2 & 9 \\
\hline Residencia de Caspe & 6 & 2 & 0 & 8 \\
\hline Colegio de San Martín (Mallorca) & 4 & 4 & 0 & 8 \\
\hline Colegio de Pollensa (Mallorca) & 5 & 3 & 0 & 8 \\
\hline Colegio de Manresa & 5 & 3 & 0 & 8 \\
\hline Colegio de Vich & 5 & 2 & 1 & 8 \\
\hline Resd. de la Sta. Cueva (Manresa) & 4 & 3 & 0 & 7 \\
\hline Colegio de Ibiza & 3 & 3 & 0 & 6 \\
\hline Residencia de San Guillermo & 2 & 3 & 0 & 5 \\
\hline TOTAL & 329 & 219 & 82 & $630^{13}$ \\
\hline
\end{tabular}

\footnotetext{
13 Al número total hay que añadirle la cifra de 30 novicios.
} 
Resultando que existían 329 sacerdotes (49,85\%), 219 coadjutores $(33,18 \%), 82$ escolares $(12,42 \%)$ y 30 novicios $(4,55 \%)$.

Sin embargo, debemos tener en cuenta algunos casos peculiares. En primer lugar, merece una especial mención el caso del sacerdote Jerónimo Julián. En la tabla y gráfico superiores lo hemos incluido como sacerdote del Colegio de Caspe, según la información que nos ofrece ARSI, Hispaniae, 147. No obstante, en otras fuentes, como AMAAEE, Santa Sede, leg. 550 se considera que este individuo pertenece al colegio de Zaragoza. ${ }^{14}$ Un caso parecido al que sucede con el sacerdote Gregorio Garcés, a quien hemos considerado como miembro del colegio de Tarazona, según la información proporcionada por ARSI, Hispaniae, 147 y AHSIC, EX. 22, pese a que AMAAEE, Santa Sede, leg. 550 lo considera como sacerdote del Colegio de Zaragoza. ${ }^{15}$ Estos dos ejemplos constituyen un claro indicativo de la dificultad para ubicar a cada religioso en su colegio, a la que hemos aludido anteriormente.

De la misma manera, hemos encontrado situaciones en las que determinados jesuitas que fueron expulsados como miembros de una provincia concreta cambiaron de provincia durante el exilio. Pero seamos más específicos; nos referimos a casos como el del estudiante Miguel Monzón, al que hemos registrado como miembro del Colegio de Urgel, que en el primer trimestre del año 1772 pasó a la provincia de Chile y se integró dentro del grupo conformado por los miembros del Colegio Máximo de San Miguel de la ciudad de Santiago de Chile. Otros casos similares fueron los del sacerdote Joaquín Carnicer, del colegio de Zaragoza, que se agregó a la provincia de Filipinas en el segundo trimestre de 1772; el coadjutor Mariano Balaguer, del colegio de Huesca, que se agregó a la provincia de Santa Fe también en el segundo trimestre de 1772, o el caso del coadjutor José Baldó, del colegio de Gerona, que se incorporó con los miembros de la provincia de México en la misma fecha que los dos últimos casos.

Como puede imaginarse, el movimiento de los jesuitas de la Provincia de Aragón no iba únicamente en una sola dirección, sino que también podía acontecer el caso contrario al que hemos relatado, es decir, que algún miembro de otra provincia pasara a integrar el conjunto de los jesuitas de la provincia de Aragón, como fue el caso del sacerdote Manuel del Barrio, miembro del Colegio de Sanlúcar de Barrameda, en la provincia de Andalucía, que se incorporó a la provincia de Aragón en el segundo trimestre de 1772.

14 Jerónimo Julián era natural de San Martín del Río (Teruel), donde nació el 2 de octubre de 1730. Ingresó en la Compañía de Jesús el 8 de agosto de 1746, en la provincia de Aragón. En 1767 embarcó en Salou en la saetía "Nuestra Señora del Buen Viaje", llegando a Bonifacio, en Córcega, el 28 de agosto. Posteriormente, en Italia, se estableció en la legación de Ferrara, con el resto de los miembros de la Provincia de Aragón. Precisamente, su muerte tendría lugar allí, en Ferrara, el 6 de diciembre de 1790.

15 Gregorio Garcés era natural de Hecho (Huesca), donde nació el 13 de marzo de 1733. Ingresó en la Compañía de Jesús con la provincia de Aragón el 14 de septiembre de 1749. En 1767 embarcó en Salou en la saetía "Nuestra Señora del Buen Viaje", llegando a Bonifacio (Córcega) el 28 de agosto de ese año. Se estableció en la legación de Ferrara, con el resto de miembros de la Provincia de Aragón. Consiguió el premio de la pensión doble por su obra de 1791 Fundamento del vigor y elegancia de la lengua castellana, expuesto en el propio y vario uso de sus partículas. Falleció en Roma el 16 de de julio de 1805.
Casos similares fueron los del sacerdote Juan Díaz, perteneciente al colegio de Honda, en la provincia de Santa Fe; el sacerdote Francisco Miguel, del colegio de Santiago en la provincia de Paraguay; o el sacerdote Domingo Roca, del colegio de Salta, también en la provincia de Paraguay, que se unieron a la de Aragón en el segundo trimestre de 1772. ${ }^{16}$

\section{JESUITAS DEPOSITADOS EN ESPAÑA}

A pesar de que las instrucciones dadas a los jueces comisionados en la expulsión manifestaban claramente la necesidad del exilio de todos los miembros de la Compañía de Jesús, se indicaba que en el caso de encontrar viejos de edad muy crecida o enfermos que no sea posible remover en el momento, habría que esperar hasta tiempo más benigno o a que su enfermedad se decida. ${ }^{17}$ Esto significaba que no todos los religiosos abandonarían el reino en el mismo momento, pues los que fuesen más ancianos y achacosos o los que a causa de una enfermedad puntual no pudieran ser desplazados deberían permanecer en el país hasta que se diesen las condiciones para su traslado, pues pese a la dureza del decreto de extrañamiento, Carlos III los seguía considerando súbditos, y en la reglamentación generada para los comisionados se insistía en que los jesuitas tenían que ser tratados con toda decencia. No debemos olvidar el caso de los procuradores de cada colegio, es decir, el de los religiosos que se encargaban de desempeñar las tareas de contabilidad en cada residencia, quienes no saldrían hacia los Estados Pontificios hasta dos meses después que el resto de sus hermanos, pues se consideraba que en ese tiempo debían poner al día a los jueces comisionados para cada localidad de las cuentas del colegio respectivo (entradas y salidas de capital y demás aspectos contables). En efecto, los procuradores saldrían hacia el exilio desde Cartagena en octubre de 1767 para reunirse con el resto de los miembros de la Compañía de Jesús.

Pero volviendo al caso de los ignacianos depositados en España, éstos debían ser trasladados a los conventos de otras órdenes religiosas que se encontrasen más cercanos, donde tendrían que permanecer, según estas estrictas órdenes, sin comunicación con el exterior.

El número de jesuitas de la provincia de Aragón que permanecieron en la península por no ser recomendable su embarque hacia los Estados Pontificios debido a su avanzada edad o a su incapacidad física o mental, no fue muy elevado, ya que estaría en torno a la decena, como recogemos en la tabla que incluimos a continuación:

16 En el caso de estos dos últimos, su incorporación a la Provincia de Aragón podría deberse a que eran naturales de esa zona geográfica: Francisco Miguel nació en L'Arboç (Tarragona) y Domingo Roca era natural de Barcelona.

17 "Instruccion de lo que deberan executar los comisionados para el extrañamiento y ocupacion de bienes y haciendas de los jesuitas en estos reynos de España e islas adjacentes, en conformidad de lo resuelto por SM", en Coleccion general de las providencias hasta aqui tomadas sobre el estrañamiento y ocupacion de temporalidades de los regulares de la Compañia, que existian en los Dominios de S.M. de España, Indias, e Islas Filipinas á consequencia del Real Decreto de 27 de Febrero y Pramática-Sancion de 2 de abril de 1767, parte primera, IV: 8-14. 
Jesuitas de la Provincia de ARAgón depositados EN ESPAÑA EN $1767^{18}$

- Francisco Mateo, sacerdote del Colegio de Alagón, quedó depositado en el convento de los agustinos descalzos de Alagón, donde falleció el 20 de abril de 1767.

- Manuel García, coadjutor del Colegio de Alagón, fue depositado en el convento de San Francisco de Zaragoza, por asmático, y allí falleció el 12 de marzo de 1774.

- Antonio Juan, sacerdote del colegio de Alicante, permaneció depositado, por demente, en el Hospital General de Valencia.

- Manuel Torres, sacerdote del Colegio de Belén de Barcelona, quedó depositado, por demente, en el Hospital de San Severo de Barcelona.

- Paulino Galcerán, coadjutor del Colegio de Belén de Barcelona, fue depositado, por viejo y tullido, en el convento de San Francisco de Barcelona, donde falleció el 12 de enero de 1768.

- Juan Enguita, coadjutor del Colegio de Graus, permaneció depositado, por enfermedad crónica, en el Convento de la Merced de Tarragona, donde falleció el 29 de noviembre de 1774

- Miguel Roca, coadjutor del Colegio de Montesión en Mallorca, quedó depositado en el Convento de los Capuchinos de Palma de Mallorca, donde falleció el 10 de mayo de 1770.

- Juan Mógica, coadjutor del Colegio de San Pablo de Valencia, fue depositado, por demente y anciano, en el Convento de la Merced de Zaragoza, donde falleció en 1770.

- José Pons, coadjutor del Colegio de Zaragoza, permaneció depositado en el Convento de San Francisco de esa ciudad, donde falleció el 18 de abril de 1774.

- José Estela, ${ }^{19}$ sacerdote del Colegio de Zaragoza, quedó depositado en el Convento de San Francisco de esa ciudad, donde falleció el 18 de abril de $1774 .^{20}$

Según las cifras de la Depositaría general de temporalidades, ${ }^{21}$ a cuyo frente se encontraba Juan Antonio Archimbaud y Solano, el número total de jesuitas que quedaron depositados en la península, a causa de diferentes motivos, fue de 77 religiosos (64 sacerdotes, 1 escolar y 12 coadjutores), si bien pensamos que se trata de una cifra bastante discutible, pues sólo refiere a dos jesuitas de la Provincia de Aragón, cuando hemos localizado al menos diez, tal y como hemos indicado en la tabla precedente.

\section{JESUITAS FALLECIDOS}

En el periodo comprendido entre 1767 y 1774, es decir, entre el momento de la expulsión de la Compañía de Jesús y el año siguiente a su extinción canónica, fallecieron 98 jesuitas de la que fuera Provincia de Aragón (47 sacerdotes, 46 coadjutores y 7 escolares). Si prestamos atención al gráfico siguiente observaremos los colegios a los que pertenecían los fallecidos:

18 En la elaboración de esta tabla hemos tomado como principal referencia AHSIC, EX, 22.

19 En ocasiones aparece como José Stela o como José Estella.

20 Según ARSI, Hispaniae, 147, su muerte tuvo lugar el 18 de junio de 1771.

21 Martínez Tornero, C. A. 2010.
JeSUITAS DE LA PROVINCIA DE ARAgón FALLECIDOS ENTRE $1767-1774 .^{22}$

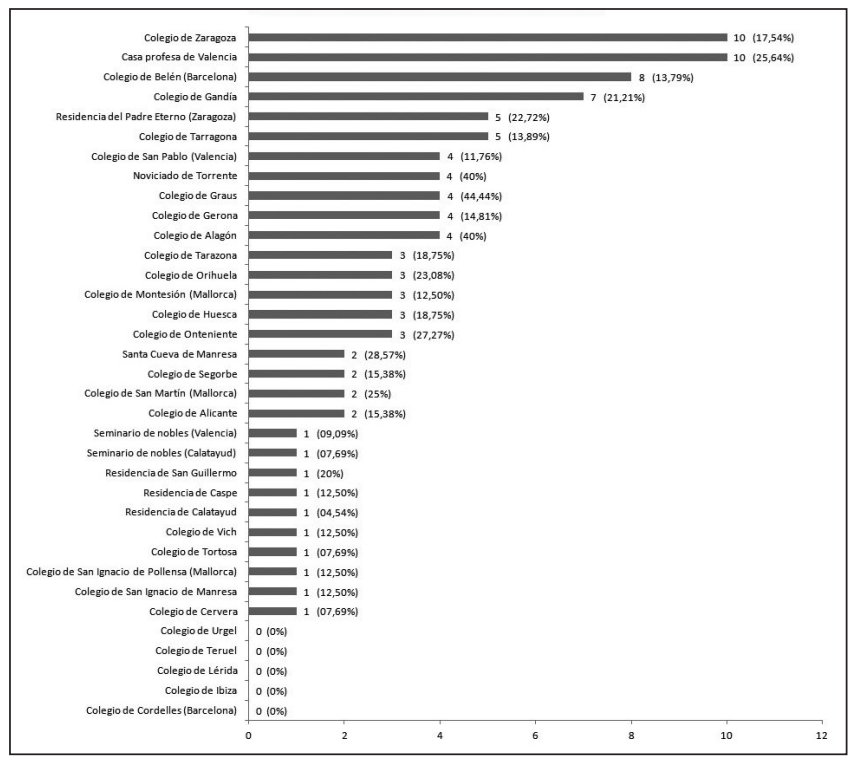

La casa profesa de Valencia, el Colegio de Zaragoza y el Colegio de Belén de Barcelona serían los colegios que perdieron un mayor número de religiosos; una situación que no debe resultar extraña si pensamos que estas residencias eran las más concurridas. Más significativas fueron las bajas de los colegios de Graus, Torrente y Alagón, que perdieron a casi la mitad de sus miembros.

Si atendemos al año de defunción de estos religiosos obtendremos el gráfico siguiente:

\section{JESUITAS FALLECIDOS ENTRE 1767-1774 (POR AÑOS) ${ }^{23}$}

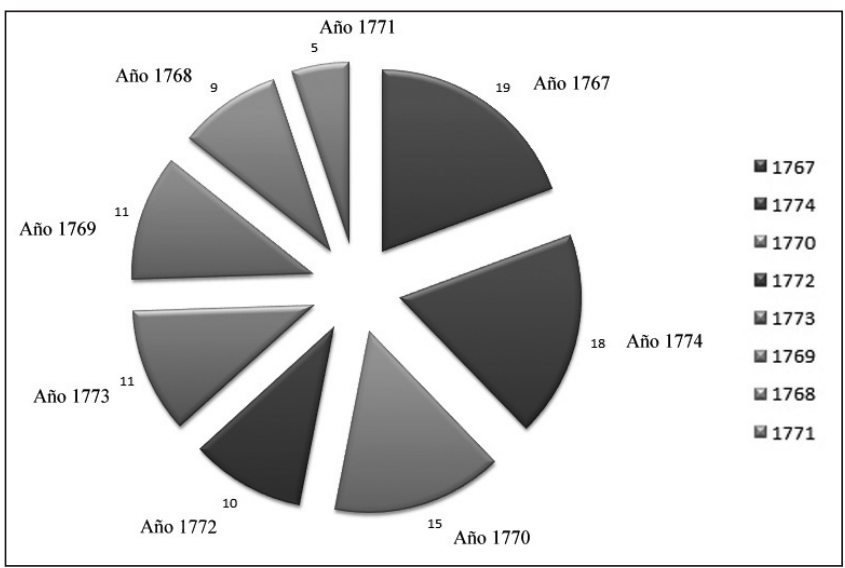

22 Fuente: Elaboración propia a partir de la información contenida en AHSIC, EX. 22 fols. 81-89; ARSI, Hispaniae, 147; ARSI, Hispaniae, 149 y AMAAEE, Santa Sede, leg. 550.

23 Los datos a partir de los cuales hemos configurado este gráfico proceden de AHSIC, EX. 22 fols. 81-89; ARSI, Hispaniae, 147; ARSI, Hispaniae, 149 y AMAAEE, Santa Sede, leg. 550. No obstante, otras fuentes como ARSI, Aragoniae, 18, ofrecen una cifra distinta de jesuitas muertos entre los años 1767-1769. De esta manera, para el año 1767 habla de 16 fallecidos en lugar de los 19 que hemos recogido; para 1768 se indica que habrían fallecido 12 religiosos, en vez de los 9 que hemos contabilizado; finalmente, en 1769 se señala la muerte de 13 jesuitas en lugar de los 11 registrados en el gráfico. En resumen, ARSI, Aragoniae, 18 señala 41 jesuitas muertos para el periodo 1767-1769, en vez de los 39 que hemos indicado en el gráfico. Las cifras serían coincidentes para el año 1770 y no se ofrecen más datos para los años posteriores. 
A la luz de los datos presentados, se observa que el momento de mayor mortalidad se registró en 1767 , el año de la expulsión, en el que se llevaron a cabo los viajes en barco más duros y la llegada a la isla de Córcega (donde se estaba librando una guerra civil entonces), siendo un periodo en el que los momentos de nerviosismo e inseguridad para estos religiosos fueron muy intensos. El segundo año con un mayor índice de mortalidad fue 1774 , el año posterior a la extinción de la Compañía, donde nuevamente los índices de estrés e incertidumbre volvieron a crecer.

\section{JESUITAS SECULARIZADOS}

La cuestión de la secularización de los expulsos fue uno de los capítulos que más preocupó a las autoridades madrileñas, ya que consideraban esta materia de una gran importancia, debido a que pensaban que mediante las secularizaciones se favorecía la quiebra de la cohesión jesuítica, o en palabras de Campomanes, su fanatismo y unión, lo cual era prioritario para los intereses del Estado. Debemos destacar que desde que se produce la expulsión de la Compañía de Jesús, los religiosos intentaron en todo momento preservar el sentido de comunidad en esas condiciones adversas, subrayando la identidad de grupo y la conciencia de pertenecer a un único cuerpo social como método para poder resistir a las adversidades políticas. Por este motivo, los ministros españoles remitieron normas muy precisas a los comisarios reales, encargados de la vigilancia y pago de la pensión a los expulsos, con el fin de que fomentaran las deserciones y rompiesen la unidad de los religiosos. ${ }^{24}$

Las motivaciones para la secularización eran fundamentalmente dos: el deseo de regresar a España y la intención de escapar de las precarias condiciones que los jesuitas experimentaban en la isla de Córcega mientras vivieron allí (problemas de alojamiento, escasez de víveres...). Además, en ese fomento de las secularizaciones desde el gobierno se concedían tres ventajas a quienes decidiesen abandonar la Compañía: un anticipo de la pensión de entre tres y seis meses, una ayuda de costa de 30 pesos con los que afrontar los gastos del viaje desde Córcega a los Estados Pontificios o al territorio italiano elegido ${ }^{25} \mathrm{y}$, desde enero de 1768 , otro suplemento de 30 pesos para gastos de vestuario. ${ }^{26}$

La secularización se lograba utilizando una de las dos vías posibles: solicitándola al General de la Compañía o haciéndolo en la Penitenciaría de Roma. No obstante, las autoridades españolas sólo aceptaban las dimisorias obtenidas del cardenal penitenciario, ya que la autoridad del General no era reconocida.

A continuación vamos a tratar de ofrecer algunas cifras en relación al número de jesuitas secularizados. ${ }^{27}$ Siguiendo

24 Fernández Arrillaga, I. 2003. "Entre el repudio y la sospecha: los jesuitas secularizados". Revista de Historia Moderna: anales de la Universidad de Alicante 21: 349-364.

25 Este subsidio quedaría reducido a 20 pesos por la resolución del Consejo Extraordinario de 7 de mayo de 1769. AGS, Gracia y Justicia, leg. 669, Grimaldi a Roda, 9 de junio de 1769.

26 Giménez López, E. y Martínez Gomis, M. 1997.

27 Para ello, hemos tomado como principal referente la fuente ARSI, Hispaniae, 150. Sin embargo, hemos de hacer constar una dificultad que las fechas ofrecidas por las diversas fuentes consultadas, se puede comprobar que el mayor número de jesuitas secularizados tiene lugar entre 1767 y 1768, es decir, en los primeros años de la expulsión y estancia en Córcega. Después de ese momento, para la provincia de Aragón, se observa un acusado descenso en el número de secularizaciones, que se mantendrá bastante estable y reducido en el periodo 1769-1773.

La cifra total de jesuitas de la Provincia de Aragón secularizados se situaría alrededor de los 63 religiosos.

SECULARIZADOS POR COLEGIOS $1767-1773^{28}$

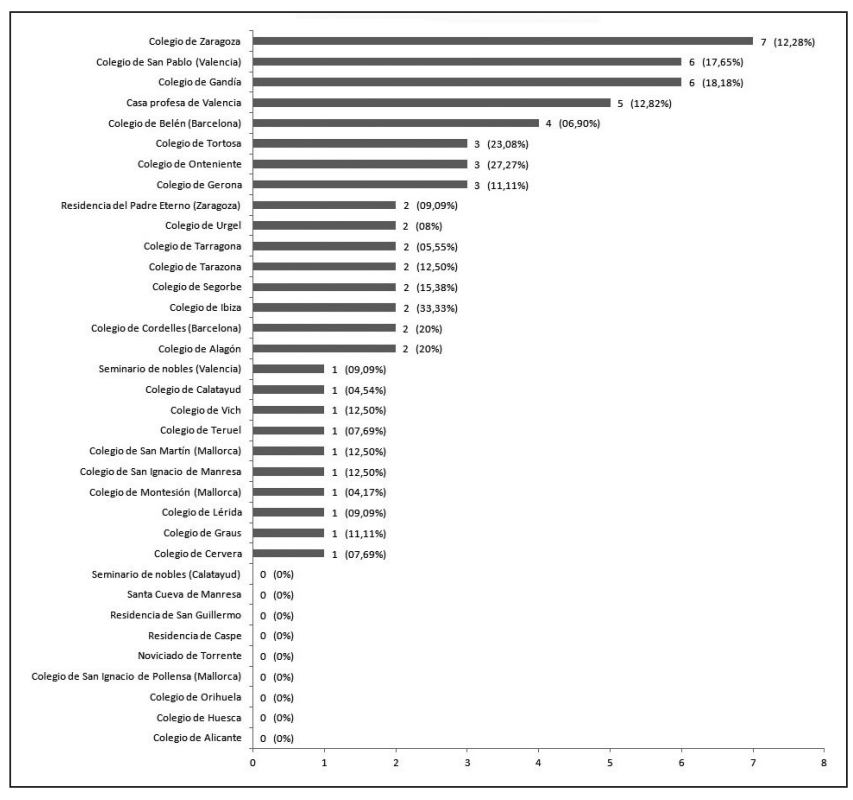

El colegio en el que se produjo un mayor número de secularizaciones fue el de Zaragoza, que era uno de los colegios más poblados en 1767 . Un caso similar al de colegios como el de San Pablo de Valencia, el de Gandía o la Casa profesa también de Valencia, que se sitúan en los primeros puestos de este gráfico, pues eran residencias de las más concurridas.

Por otra parte, sorprende que el colegio con un mayor número de residentes, el de Belén de Barcelona, no sea el lugar en el que abandona la Compañía de Jesús un número mayor de religiosos, sino que se sitúa en el quinto puesto. Algo similar a lo que sucede con el Colegio de Tarragona, que es el cuarto en cuanto al número de habitadores, pero el undécimo en lo que se refiere a las secularizaciones.

se observa en el análisis de la documentación. Entre toda la información examinada, hemos podido comprobar un cierto baile de fechas en cuanto al momento en el que se secularizan determinados individuos. Tómese por ejemplo el caso del sacerdote de la Casa Profesa de Valencia Joaquín López. Según la fuente ARSI, Hispaniae, 147, su secularización se produjo el 6 de septiembre de 1768. No obstante, la relación que aparece en ARSI, Hispaniae, 150, sitúa su secularización el 6 de julio de 1773, es decir, cinco años después. Incluso existen otras fuentes que hablan del 5 de enero de 1769. El caso de Joaquín López no es único. Encontramos con mucha frecuencia fechas de secularización para un mismo individuo que difieren en días, en meses o incluso en años, dependiendo de la documentación consultada. Este tipo de información la podemos encontrar en fuentes como ARSI, Hispaniae, 147; ARSI, Hispaniae, 150; ARSI, Aragoniae, 18; AMAAEE, Santa Sede, leg. 551...

28 Elaboración propia: creado a partir de la información contenida en ARSI, Hispaniae, 150, para la provincia de Aragón. 
Destaca el hecho de que no se secularizase ningún religioso en Huesca, un colegio habitado por un número de jesuitas similar al de otros lugares como Tarazona, donde se produjeron dos secularizaciones, y con un número de residentes superior a otras residencias como las de Tortosa (tres secularizaciones), Segorbe (dos secularizaciones) o Teruel (una secularización).

Finalmente, los centros con un porcentaje de secularizados mayor fueron el Colegio de Ibiza (33,33\%) y el Colegio de Onteniente $(27,27 \%)$.

\section{JESUITAS SUSPENDIDOS DE PENSIÓN}

La Pragmática Sanción de 2 de abril de 1767 reconocía un subsidio que habría que pagar de manera vitalicia a todos los miembros expulsos de los dominios de Carlos III. Esta gratificación económica, que procedía del conjunto de los bienes de la Compañía de Jesús, se entregaba con una finalidad doble: en primer lugar, se pretendía que el Papa no rechazase a los más de cinco mil jesuitas que se le enviaban desde el reino hispano alegando motivos económicos, pues los Estados Pontificios ya se estaban haciendo cargo de la manutención de los jesuitas portugueses, los cuales habían sido expulsados sin ningún tipo de subvención. ${ }^{29}$ En segundo lugar, la pensión se convirtió en una herramienta con una clara finalidad política, al castigar con la pérdida de este subsidio a los religiosos que realizasen escritos en contra de la monarquía hispana; asimismo, mediada la década de 1770 se considera que existe una campaña a nivel europeo de descrédito de la cultura española, pensando las autoridades que los ignacianos podrían contrarrestar esa situación desfavorable para los intereses hispanos, de manera que se deciden a premiar con pensiones dobles o incluso triples a aquellos que hiciesen labores propagandísticas y favorables al Estado. ${ }^{30}$

En concreto, la pensión vitalicia consistía en el pago de 100 pesos anuales de a 15 reales de vellón para los sacerdotes, mientras que los coadjutores recibían una retribución anual de 90 pesos. Los novicios, en principio, no percibirían esta gratificación económica, al considerarse que seguían a los jesuitas libremente, ya que se les dio la opción de abandonarles e ingresar en otra Orden. Sin embargo, una vez llevada a cabo la extinción de la Compañía de Jesús, se amplió el disfrute de la pensión a los novicios, gracias a un despacho firmado por Carlos III el 2 de mayo de $1774 .{ }^{31}$

A continuación, incluimos la relación de los religiosos de la Provincia de Aragón que fueron novicios en el momento del destierro y que comenzaron a cobrar la pensión a partir del 21 de junio de 1774:

29 Sobre la expulsión de los jesuitas en Portugal véase: Lopes, A 2002. Enigma Pombal. Lisboa: Roma Editora; Ferrao, A. 1923-1924. “O marquês de Pombal e a expulsao dos jesuitas". Boletin da Segunda Clase da Academia das Sciencias de Lisboa, vol. XVIII: 253-626.

30 Guasti, N. 2006. L'esilio italiano dei gesuiti spagnoli. Identità, controllo sociale e pratiche culturali. Roma: Ed. di Storia e Letteratura.

31 Fernández Arrillaga, I. 2004. El destierro de los jesuitas casteIlanos (1767-1815), Valladolid: Junta de Castilla y León, Consejería de Cultura y Turismo.
NOVICIOS DE LA PROVINCIA DE ARAGÓN QUE EMPEZARON A COBRAR LA PENSIÓN A PARTIR DE $1774^{32}$
- José García
- Agustín Monzón
- Gaspar Sánchez
- Francisco Javier Giner
- Francisco Catalá
- Mariano Lázaro
- Antonio Alemany
- Vicente Suárez
- José Ferrer
- Mariano Llorente
- Juan Roca
- Antonio Puget
- Antonio Prieto
- Antonio Pinazo
- Narciso Roger
- Manuel Gutiérrez
- José Puchol
- Pablo Milá
- Ramón Pla
- Peregrino Anoll
- Miguel Lizabe
- Hilario Comamala
- Joaquín Trullench
- Antonio de Antonio

En el periodo que estamos estudiando (1767-1774) no se concedió a ningún jesuita el premio de la doble pensión, como sí sucederá más adelante. Sirva de ejemplo el caso de Francisco Javier Llampillas, ${ }^{33}$ sacerdote y catedrático de Filosofía del Colegio de Belén de Barcelona, que residiendo en Génova en 1778 recibió la gracia de doble pensión, la cual le fue concedida en atención a sus méritos literarios. ${ }^{34}$

No obstante, sí que se llegó a castigar a determinados jesuitas en base a su comportamiento, poco adecuado a las directrices de la monarquía hispana. En concreto, nos referimos al estudiante del colegio de Gandía José Ferrándiz, que se secularizó en 1767. Ferrándiz fue suspendido del goce de su pensión desde el 6 de mayo de 1771 por haber celebrado la primera misa en una de las capillas privadas de la que fue Casa Profesa del Jesús.

También hemos detectado la suspensión de la pensión a los jesuitas Diego Adam, sacerdote de la Casa profesa de Valencia, y a Juan Luis Colomb, sacerdote del Colegio de Belén de Barcelona; en ambos casos, el motivo por el que se tomó esta severa disposición con ellos fue porque se convirtieron en prófugos.

Otros sacerdotes que huyeron de Córcega y que no aparecen reflejados en los listados que se forman para controlar la recogida de la pensión de los miembros de la Provincia de Aragón, establecidos en la legación de Ferrara, ${ }^{35}$ son los siguientes:

- Sebastián Torrente, coadjutor del Colegio de Zaragoza.

- Ignacio Sánchez, coadjutor del Colegio de Zaragoza.

- José Manuel Vidal, sacerdote del Colegio de Gandía. EX. 22.

Cuadro elaborado a partir de la información contenida en AHSIC,

33 Francisco Javier Llampillas era natural de Mataró (Barcelona), donde nació el 1 de diciembre de 1731. Ingresó en la Compañía de Jesús el 8 de junio de 1747. Fue embarcado rumbo al exilio en Salou en la saetía "San Antonio" en 1767, llegando a Bonifacio (Córcega) el 28 de agosto de ese año. En un primer momento se estableció en la legación de Ferrara, con el resto de los miembros de la Provincia de Aragón, pero posteriormente, a partir de 1774, se trasladaría a Génova, donde recibió la gracia de la doble pensión por su trabajo literario. Su muerte tendría lugar en Génova en noviembre de 1798.

34 AGS, Estado, Leg. 5.041. Véase también Giménez López, E. y Pradells Nadal, J. 1996. "Los jesuitas expulsos en el viaje a Italia de Nicolás Rodríguez Lasso (1788-1789)". Revista de Historia Moderna, 15: 233-253.

35 Estos listados se pueden consultar en AMAAEE, Santa Sede, leg. 551. 
- Mariano Miguel, coadjutor del Colegio de Gandía.

- Juan Francisco Rouvelet, sacerdote del Colegio de Belén de Barcelona.

- Antonio Martel, estudiante del Colegio de Calatayud.

- Antonio García, coadjutor de la Casa profesa de Valencia.

- Antonio Eximeno, sacerdote de la provincia de Aragón, que en el momento de la expulsión se encontraba como profesor de matemáticas en el Colegio Militar de Segovia. ${ }^{36}$

Tenemos noticia de la secularización de casi todos ellos, y algunos incluso llegarían a casarse, como Antonio García, Mariano Miguel e Ignacio Sánchez. ${ }^{37}$

\section{ESTADO DE LA COMPAÑÍA DE JESÚS EN 1774}

Atendiendo a toda la información aportada, estamos en condiciones de ofrecer una buena aproximación en lo que se refiere al estado de la Provincia de Aragón de la Compañía de Jesús el año posterior a su extinción.

En el gráfico siguiente podemos comprobar la evolución del número de jesuitas en el periodo que interesa a nuestro estudio, entre 1767 y 1774 . En color oscuro se expresa el número de los jesuitas existentes en 1767 y en color claro se muestra el conjunto de religiosos que continuaban en 1774.

\section{EVOLUCIÓN DEL NÚMERO DE JESUITAS EN EL PERIODO 1767-1774}

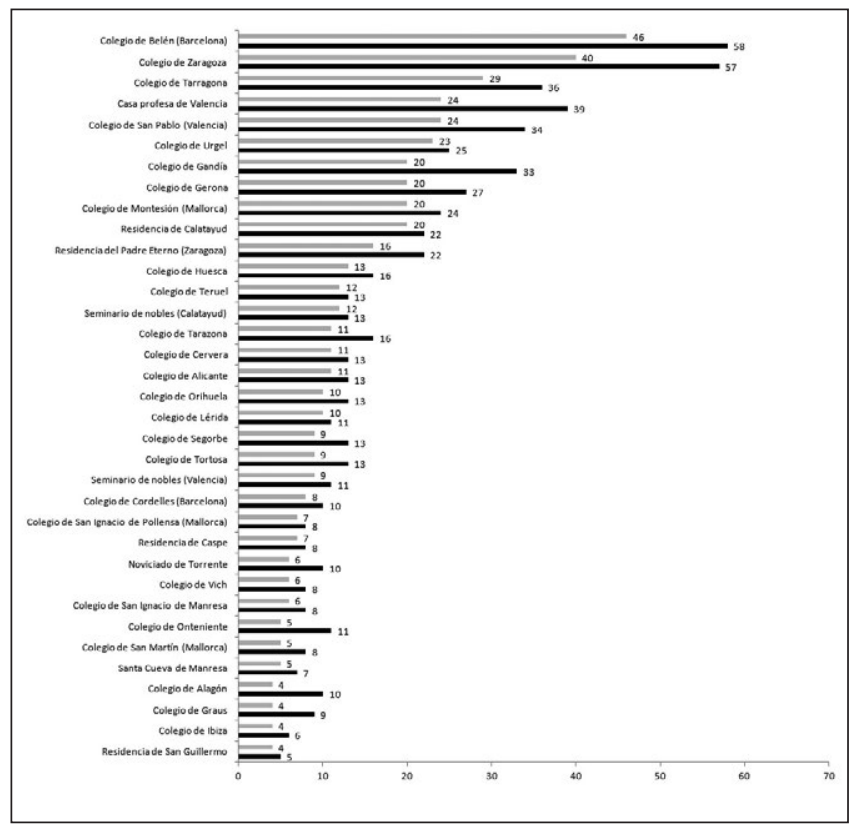

${ }^{36}$ Sobre este jesuita véase Picó Pascual, M. A. 2003. El padre José Antonio Eximeno Pujades. Valencia: Edicions Alfons el Magnànim; Bono Guardiola, M. J. 1997. “Una sátira filosófica: ‘El espíritu de Maquiavelo de Antonio Eximeno'”, en E. Giménez (ed.), Expulsión y exilio de los jesuitas españoles: 331-345. Alicante: Publicaciones de la Universidad de Alicante.

37 Antonio García se casó en Roma, donde tuvo dos hijos: Juan y María, que fueron bautizados el 30 de mayo de 1775 y el 11 de noviembre de 1776, respectivamente. Mariano Miguel se casó con Juliana Nicolás, con la que no tuvo hijos. Ignacio Sánchez se casó en Roma y tuvo una hija, María Ángela, que fue bautizada el 2 de diciembre de 1783.

Hispania Sacra, LXVIII

137, enero-junio 2016, 343-353, ISSN: 0018-215X, doi: 10.3989/hs.2016.023
Queda claro que, por lo general, los colegios que sufrieron un número mayor de bajas fueron los que estaban habitados por un número mayor de individuos. Además del Colegio de Belén de Barcelona y el Colegio de Zaragoza, tuvo gran importancia la reducción, en lo que a número de miembros se refiere, experimentada en la Casa profesa de Valencia (15), el Colegio de Gandía (13) o el Colegio de San Pablo de Valencia (10). Sin embargo, más significativas fueron las mermas en los colegios de Alagón, Graus u Onteniente, ya que en el periodo estudiado perdieron a más de la mitad de sus integrantes ( $60 \%, 55,56 \%$ y $54,54 \%$, respectivamente).

A lo largo de estas páginas hemos tratado de reconstruir, de manera aproximada, la evolución del número de los jesuitas que integraban la Provincia de Aragón en el exilio entre dos fechas de gran importancia, como fueron el momento de la expulsión y el de la extinción de la Compañía de Jesús. De esta forma, hemos procurado clarificar, para una zona geográfica bien delimitada, un aspecto sobre el que planeaban algunas dudas, como la del número de los jesuitas que se vieron afectados por la medida de expulsión y el impacto cuantitativo que tuvo para la orden religiosa tanto el exilio como su supresión canónica.

Igualmente, hemos procurado ofrecer, para la provincia y el periodo estudiados, otros datos de importancia como el de los novicios que comenzaron a percibir la pensión vitalicia a partir de 1774, el de los religiosos que permanecieron depositados en la península a causa de su estado físico o mental, el del número de jesuitas secularizados... y asimismo, hemos señalado alguna información sobre los ignacianos que perdieron el disfrute de su subsidio económico o huyeron.

Con todo ello, consideramos que podemos obtener un retrato aproximado de la que fuera Provincia de Aragón, el año después de su extinción. 


\begin{tabular}{|c|c|c|c|}
\hline Colegio de pertenencia & Muertos & Secularizados & № de pérdidas \\
\hline Colegio de Zaragoza & 10 & 7 & $17(29,82 \%)$ \\
\hline Casa profesa de Valencia & 10 & 5 & $15(38,46 \%)$ \\
\hline Colegio de Gandía & 7 & 6 & $13(39,39 \%)$ \\
\hline Colegio de Belén (Barcelona) & 8 & 4 & $12(20,69 \%)$ \\
\hline Colegio de San Pablo (Valencia) & 4 & 6 & $10(29,41 \%)$ \\
\hline Colegio de Gerona & 4 & 3 & $7(25,93 \%)$ \\
\hline Colegio de Tarragona & 5 & 2 & $7(19,44 \%)$ \\
\hline Residencia del Padre Eterno (Zaragoza) & 5 & 2 & $6 *(10,53 \%)$ \\
\hline Colegio de Onteniente & 3 & 3 & $6(54,54 \%)$ \\
\hline Colegio de Alagón & 4 & 2 & $6(60,00 \%)$ \\
\hline Colegio de Tarazona & 3 & 2 & $5(31,25 \%)$ \\
\hline Colegio de Graus & 4 & 1 & $5(55,56 \%)$ \\
\hline Colegio de Tortosa & 1 & 3 & $4(30,77 \%)$ \\
\hline Colegio de Segorbe & 2 & 2 & $4(30,77 \%)$ \\
\hline Colegio de Montesión (Mallorca) & 3 & 1 & $4(16,67 \%)$ \\
\hline Noviciado de Torrente & 4 & 0 & $4(40,00 \%)$ \\
\hline Colegio de San Martín (Mallorca) & 2 & 1 & $3(37,50 \%)$ \\
\hline Colegio de Huesca & 3 & 0 & $3(18,75 \%)$ \\
\hline Colegio de Orihuela & 3 & 0 & $3(23,08 \%)$ \\
\hline Colegio de Cordelles (Barcelona) & 0 & 2 & $2(20,00 \%)$ \\
\hline Colegio de Ibiza & 0 & 2 & $2(33,33 \%)$ \\
\hline Colegio de Urgel & 0 & 2 & $2(08,00 \%)$ \\
\hline Colegio de Cervera & 1 & 1 & $2(15,38 \%)$ \\
\hline Colegio de San Ignacio de Manresa & 1 & 1 & $2(25,00 \%)$ \\
\hline Colegio de Vich & 1 & 1 & $2(25,00 \%)$ \\
\hline Residencia de Calatayud & 1 & 1 & $2(09,09 \%)$ \\
\hline Seminario de nobles (Valencia) & 1 & 1 & $2(18,18 \%)$ \\
\hline Colegio de Alicante & 2 & 0 & $2(15,38 \%)$ \\
\hline Santa Cueva de Manresa & 2 & 0 & $2(28,57 \%)$ \\
\hline Colegio de Lérida & 0 & 1 & $1(09,09 \%)$ \\
\hline Colegio de Teruel & 0 & 1 & $1(07,69 \%)$ \\
\hline Colegio de San Ignacio de Pollensa (Mallorca) & 1 & 0 & $1(12,50 \%)$ \\
\hline Residencia de Caspe & 1 & 0 & $1(12,50 \%)$ \\
\hline Residencia de San Guillermo & 1 & 0 & $1(20,00 \%)$ \\
\hline Seminario de nobles (Calatayud) & 1 & 0 & $1(07,69 \%)$ \\
\hline
\end{tabular}

\footnotetext{
* En el caso de la Residencia del Padre Eterno de Zaragoza, el coadjutor Joaquín Camarasa se secularizó en 1768 ó 1769 , dependiendo de la fuente, y murió en 1772; por eso, aunque indicamos que murieron cinco jesuitas y se secularizaron dos, el número total de religiosos perdidos fue de seis y no de siete, pues si indicásemos ese número estaríamos contando dos veces a Joaquín Camarasa.
} 
Anexo 1

Edad de los jesuitas de la Provincia de Aragón en 1767*

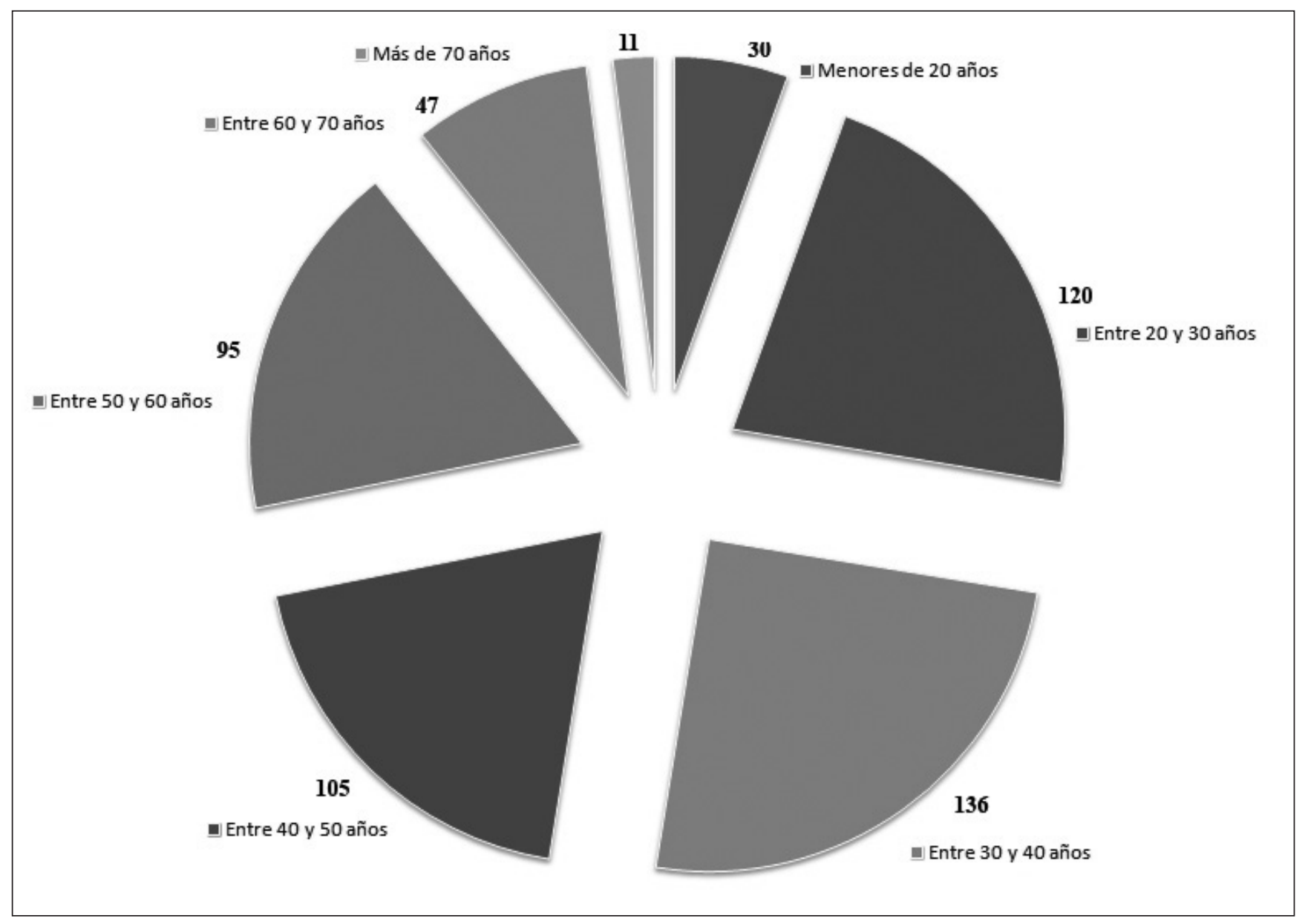

"Para la elaboración de este gráfico no hemos podido contar con los datos referentes al nacimiento de todos los expulsos pertenecientes a la Provincia de Aragón, pero sí con una buena muestra de ellos. De hecho, hemos averiguado esta información para un total de 544 religiosos, de los 660 que consideramos que formaban esta Provincia jesuita, y con esos datos hemos realizado este diagrama. 


\section{BibLIOgRAFÍA}

1767. Coleccion general de las providencias hasta aqui tomadas sobre el estrañamiento y ocupacion de temporalidades de los regulares de la Compañia, que existian en los Dominios de S.M. de España, Indias, e Islas Filipinas á consequencia del Real Decreto de 27 de Febrero y Pramática-Sancion de 2 de abril de 1767. Madrid: Imprenta Real de la Gazeta.

Bono Guardiola, M. J. 1997. “Una sátira filosófica: ‘El espíritu de Maquiavelo de Antonio Eximeno', en E. Giménez (ed.), Expulsión y exilio de los jesuitas españoles: 331-345. Alicante: Publicaciones de la Universidad de Alicante.

Egido, T. 1979. "La expulsión de los jesuitas de España”, en R. García Villoslada (coord.), Historia de la Iglesia en España, vol. IV: 745-792, Madrid: Biblioteca de Autores Cristianos.

Fernández Arrillaga, I. 2002. "Los novicios de la Compañía de Jesús: la disyuntiva ante el autoexilio y su estancia en Italia", en E. Giménez (ed.), Y en el tercero perecerán. Gloria, caída y exilio de los jesuitas españoles en el siglo XVIII: 251 278, Alicante: Publicaciones de la Universidad de Alicante.

Fernández Arrillaga, I. 2003. "Entre el repudio y la sospecha: los jesuitas secularizados". Revista de Historia Moderna: anales de la Universidad de Alicante 21: 349-364.

Fernández Arrillaga, I. 2004. El destierro de los jesuitas castellanos (1767-1815), Valladolid: Junta de Castilla y León, Consejería de Cultura y Turismo.

Ferrao, A. 1923-1924. "O marquês de Pombal e a expulsao dos jesuitas". Boletin da Segunda Clase da Academia das Sciencias de Lisboa, vol. XVIII: 253-626.

Ferrer Benimeli, J. A. 1996. La expulsión y extinción de los jesuitas según la correspondencia diplomática francesa, vol. II: 10 y 238, Zaragoza: Universidad de Zaragoza y Universidad Tachira.
Giménez López, E. y Martínez Gomis, M. 1997. “La secularización de los jesuitas expulsos (1767-1773)”, en E. Giménez (coord.), Expulsión y exilio de los jesuitas españoles: 259-304, Alicante: Publicaciones de la Universidad de Alicante.

Giménez López, E. y Pradells Nadal, J. 1996. “Los jesuitas expulsos en el viaje a Italia de Nicolás Rodríguez Lasso (1788-1789)". Revista de Historia Moderna, 15: 233-253.

Giménez López, Enrique 2002. “Portugal y España contra Roma. Los inicios del proceso de extinción de la Compañía de Jesús (1767-1769)", en E. Giménez (ed.), Y en el tercero perecerán. Gloria, caída y exilio de los jesuitas españoles en el s. XVIII: 163-193. Alicante: Universidad de Alicante.

Giménez López, Enrique 2008. Misión en Roma. Floridablanca y la extinción de los jesuitas. Murcia: Universidad de Murcia.

Giménez López, Enrique: "La extirpación de la mala doctrina: los primeros pasos para la extinción de la Compañía de Jesús", en E. Martínez Ruiz y M. de Pazzis Pi Corrales (coords.), Dogmatismo e intolerancia: 257-274. Madrid: Editorial Actas.

Guasti, N. 2006. L'esilio italiano dei gesuiti spagnoli. Identità, controllo sociale e pratiche culturali. Roma: Ed. di Storia e Letteratura.

Lopes, A. 2002. Enigma Pombal. Lisboa: Roma Editora

Martínez Tornero, C. A. 2010. Carlos III y los bienes de los jesuitas: la gestión de las temporalidades por la monarquía borbónica (1767-1815). Alicante: Publicaciones de la Universidad de Alicante.

Pastor, L. 1954. Storia dei Papi, vol. XVI, I: 754, Roma: Desclée A.C. Editori Pontifici.

Picó Pascual, M. A. 2003. El padre José Antonio Eximeno Pujades. Valencia: Edicions Alfons el Magnànim. 